\title{
Fractional Order Transient Thermoelastic Stress Analysis of a Thin Circular Sector Disk
}

\author{
Kishor R. Gaikwad*1 and Satish G. Khavale ${ }^{1}$ \\ ${ }^{1}$ P.G. Department of Mathematics, N.E.S. Science College, Nanded-431602, (M.S.), India. \\ E-mail: ${ }^{1}$ drkr.gaikwad@yahoo.in, ${ }^{2}$ khavalesatish8@gmail.com
}

Received 04 November 2020, Revised 09 August 2021, Accepted 06 September 2021

\begin{abstract}
Analysis of transient thermoelastic stress distribution of a thin circular sector disk with a time-fractional derivative of order $\alpha$ is proposed. The Neumann types of boundary conditions are used and the integral transform method and Caputo fractional derivative are used to obtain the analytical solutions of the temperature, displacement, and stresses. Numerical values of temperature, displacement, and stresses are computed for an Aluminum (pure) material and presented graphically with help of Mathcad software.
\end{abstract}

Keywords: Circular sector disk; thermal stresses; fractional order derivative; heat conduction.

\section{Introduction}

Lord et al. [1] introduced the generalized thermoelastic theory of dynamical system with one relaxation time, for the isotropic body. Green et al. [2] proposed the behavior of thermoelastic material without energy dissipation with linear and nonlinear theories. Ootao et. al. [3] solved the 3D problem for anon-homogeneous hollow circular cylinder with moving heat sources in the axial direction and it's transient thermal stresses. Ishihara et al. [4] presented the theoretical approach of thermoelastic deformation for a circular plate with a partially distributed heat supply. Sherief et. al. [5] studied the two-dimensional problem for a half-space whose surface is traction-free and subjected to the effects of heat sources is considered within the context of the theory of thermoelasticity with two relaxation times. Podlubny [6] presented the geometrical and physical interpretation of fractional integration and fractional differentiation. Povstenko [7] proposed the stresses corresponding to the fundamental solutions of a Cauchy problem for the fractional heat conduction equation in onedimensional and two dimensional cases using the Caputo fractional derivative. Sherief et. al. [8] discussed the problem of a thermoelastic half-space with a permeating substance in contact with the bounding plane in the context of the theory of generalized thermoelastic diffusion with one relaxation time. Povstenko [9] discussed the heat conduction with time and space fractional derivatives and on the theory of thermal stresses based on this equation. Sherief et. al. [10] derived the new theory of thermoelasticity using the methodology of fractional calculus and the theories of coupled thermoelasticity and of generalized thermoelasticity with one relaxation time discussed.

Gaikwad et. al. [11] studied the quasi-static thermoelastic mathematical model for infinitely long circular cylinder by using the integral transform technique. El-Karamany et. al. [12] introduced the two general models of fractional heat conduction for non-homogeneous anisotropic elastic solids and the constitutive equations for thermoelasticity theory are obtained, uniqueness and reciprocal theorems are proved and the convolution variational principle is established and used to prove a uniqueness theorem with no restriction on the elasticity or thermal conductivity tensors except for symmetry conditions. Sur et. al. [13] proposed a new theory of twotemperature generalized thermoelasticity is constructed in the context of a new consideration of heat conduction with fractional orders. Gaikwad et. al. [14] studied the nonhomogeneous heat conduction problem and its thermal deflection due to internal heat generation in a thin hollow circular disk. Gaikwad [15] analyzed the thermoelastic deformation of a thin hollow circular disk due to a partially distributed heat supply. Sur et. al. [16] proposed a new mathematical model of thermoelasticity theory in the context of a new consideration of heat conduction with fractional-order theory. A functionally graded isotropic unbounded medium is considered subjected to a periodically varying heat source in the context of spacetime non-local generalization of three-phase-lag thermoelastic model and Green-Naghdi models. Raslan [17] studied the fractional order theory of thermoelasticity to a 1D problem of an infinitely long cylindrical cavity. Raslan [18] introduced the fractional-order theory of thermoelasticity to the two-dimensional problem of a thick plate whose lower and upper surfaces are traction-free and subjected to the given axi-symmetric temperature distribution. Gaikwad [19] studied the mathematical modeling of thermoelastic problem in a circular sector disk subject to heat generation. Gaikwad [20] proposed the twodimensional study-state temperature distribution of a thin circular plate due to uniform internal energy generation. Gaikwad [21] discussed the axi-symmetric thermoelastic stress analysis of a thin circular plate due to heat generation. Gaikwad [22] studied the time-fractional heat conduction problem in a thin hollow circular disk and its thermal deflection. 
In this work, the analysis of transient thermoelastic stress distribution of a thin circular sector disk with a timefractional derivative of order $\alpha$ is proposed. The Neumann types of boundary conditions are used and the integral transform method and Caputo fractional derivative are used to obtain the analytical solutions of the temperature, displacement, and stresses. Numerical values of temperature, displacement, and stresses are computed for an Aluminum (pure) material and presented graphically with help of Mathcad software. It is believed that this particular problem has not been considered by anyone. This is a new and novel contribution to the field of thermoelasticity. The results presented here will be more useful in engineering problems particularly, in the determination of the state of strain in a thin circular disk constituting foundations of containers for hot gases or liquids, in the foundations for furnaces, etc.

\section{Formulation of the Problem}

Consider a 2D problem for a circular sector disk occupying the space $0 \leq r \leq a, 0 \leq \varphi \leq \varphi_{0} \leq 2 \pi, 0 \leq z \leq h$. A mathematical model is prepared considering nonlocal Caputo type time fractional heat conduction equation of order $\alpha$ for a thin circular disk.

"The definition of Caputo type fractional derivative given by [23]

$$
D^{\alpha} f(t)=\left\{\begin{array}{cl}
\frac{1}{\sqrt{n-\alpha}} \int_{0}^{t} \frac{f^{n}(\tau)}{(t-\tau)^{\alpha+1-n}} d \tau, & n-1<\alpha<n ; \\
\frac{d f(t)}{d t}, & n=1
\end{array}\right.
$$

For finding the Laplace transform, the Caputo derivative requires information of the initial values of the function $f(t)$ and its integer derivative of the order $k=1,2, \ldots, n-1$

$$
L\left\{D^{\alpha} f(t) ; s\right\}=s^{\alpha} F(s)-\sum_{k=0}^{n-1} s^{\alpha-k-1} f^{(k)}(0), \quad n-1<\alpha<n
$$

Also, the definition of Riemann-Liouville fractional derivative given by [23]

${ }_{a} D_{t}^{\alpha}=\left(\frac{d}{d t}\right)^{n} \int_{a}^{t}(t-\tau)^{n-\alpha} f(\tau) d \tau, \quad n-1<\alpha<n$

The temperature of the circular sector disk $T(r, \varphi, z, t)$ at time $t$ satisfying the time fractional differential equation,

$$
\frac{\partial^{2} T}{\partial r^{2}}+\frac{1}{r} \frac{\partial T}{\partial r}+\frac{1}{r^{2}} \frac{\partial^{2} T}{\partial \varphi^{2}}+\frac{\partial^{2} T}{\partial z^{2}}=\frac{1}{c} \frac{\partial^{\alpha} T}{\partial t^{\alpha}}
$$

in $0 \leq r \leq a, 0 \leq \varphi \leq \varphi_{0} \leq 2 \pi, 0 \leq z \leq h$, for $\mathrm{t}>0$,

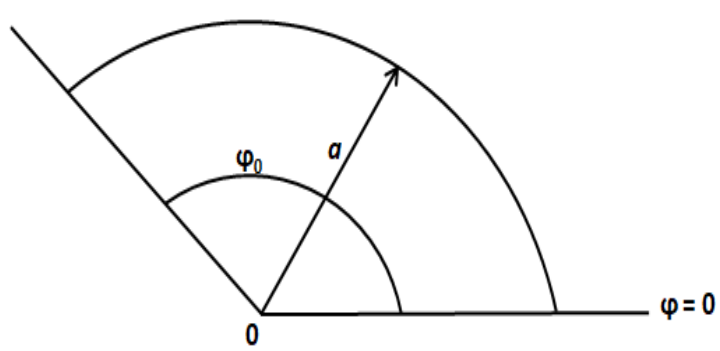

Figure 1. Geometrical representation of the problem. with the boundary conditions,

$k D_{R L}^{I-\alpha} \frac{\partial T}{\partial r}=f_{1}(\varphi, z, t), \quad$ at $r=a$, for $\mathrm{t}>0$

$k D_{R L}^{I-\alpha} \frac{\partial T}{\partial r}=f_{2}(r, z, t), \quad$ at $\varphi=0$, for $\mathrm{t}>0$

$k D_{R L}^{I-\alpha} \frac{\partial T}{\partial r}=f_{3}(r, z, t), \quad$ at $\varphi=\varphi_{0}, \quad$ for $\mathrm{t}>0$

$k D_{R L}^{I-\alpha} \frac{\partial T}{\partial r}=f_{4}(r, \varphi, t), \quad$ at $z=0$, for $\mathrm{t}>0$

$k D_{R L}^{I-\alpha} \frac{\partial T}{\partial r}=f_{5}(r, \varphi, t), \quad$ at $z=h$, for $\mathrm{t}>0$

where, $k$ is the thermal conductivity, and $D_{R L}^{\alpha} T(r, \varphi, z, t)$ for $\alpha>0$ is the Riemann-Liouville fractional integral $I^{\alpha} T(r, \varphi, z, t)$ and initial conditions,

$T=0, \quad$ at $\quad t=0, \quad 0<\alpha<1$,

$\frac{\partial T}{\partial t}=0, \quad$ at $t=0, \quad 1<\alpha<2$,

Following Gaikwad [20], we assumed that for small thickness $h$ the circular disk is in a plane state of stress. In fact, "the smaller the thickness of the hollow disk compared to its diameter, the nearer to a plane state of stress is the actual state". The displacement equations:

$$
U_{i, k k}+\left(\frac{1+v}{1-v}\right) e_{, i}=2\left(\frac{1+v}{1-v}\right) a_{t} T_{, i}, e=U_{k, k ;}, k, i=1,2
$$

Introducing

$U_{i}=\psi_{, i}, i=1,2$,

we have

$\nabla^{2} \psi=(1+v) a_{t} T$

$\nabla^{2}=\frac{\partial^{2}}{\partial x^{2}}+\frac{\partial^{2}}{\partial y^{2}}$

$\sigma_{i j}=2 \mu\left(\psi_{, i j}-\delta_{i j} \psi_{, k k}\right), \quad i, j, k=1,2$

The displacement potential function $\psi(r, \varphi, z, t)$ is expressed as:

$$
\frac{\partial^{2} \psi}{\partial r^{2}}+\frac{1}{r} \frac{\partial^{2} \psi}{\partial y^{2}}=(1+v) a_{t} T
$$

with $\frac{\partial \psi}{\partial r}=0$ at $r=a$ for all time $t$.

Initially $T=\psi=0$ at $t=0$

The stresses function $\sigma_{r r}$ and $\sigma_{\theta \theta}$ are: 
$\sigma_{r r}=\frac{-2 \mu}{r} \frac{\partial \psi}{\partial r}$

$\sigma_{\theta \theta}=-2 \mu \frac{\partial^{2} \psi}{\partial r^{2}}$

The traction-free boundary conditions as:

$$
\begin{aligned}
& \sigma_{r r}=\sigma_{r \varphi}=0 \text { for } r=a, 0 \leq \varphi<\varphi_{0}, \mathrm{t}>0 \\
& \sigma_{\varphi \varphi}=\sigma_{r \varphi}=0 \text { for } 0 \leq r<a, \varphi=0, \mathrm{t}>0 \\
& \sigma_{\varphi \varphi}=\sigma_{r \varphi}=0 \text { for } 0 \leq r<a, \varphi=\varphi_{0}, \mathrm{t}>0
\end{aligned}
$$

Equations (4) to (23) constitute the problem formulation under consideration.

\section{Solution of the Heat Conduction Problem}

To obtain the expression for temperature function $T(r, \varphi, z, t)$; firstly we define the finite Fourier transform and its inverse transform over the variable $z$ in the range $0 \leq z \leq h$ defined in [24] as

$$
\begin{aligned}
& \bar{T}\left(r, \varphi, \eta_{p}, t\right)=\int_{z^{\prime}=0}^{h} K\left(\eta_{p}, z^{\prime}\right) T\left(r, \varphi, z^{\prime}, t\right) d z^{\prime} \\
& T(r, \varphi, z, t)=\sum_{n=1}^{\infty} K\left(\eta_{p}, z\right) \bar{T}\left(r, \varphi, \eta_{p}, t\right)
\end{aligned}
$$

where

$$
K\left(\eta_{p}, z\right)=\sqrt{\frac{2}{h}} \cos \left(\eta_{p} z\right)
$$

and $\eta_{1}, \eta_{2} \ldots$ are the positive roots of the transcendental equation

$$
\begin{aligned}
& \sin \left(\eta_{p} h\right)=0, \quad p=1,2,3 \ldots \\
& \text { i.e. } \quad \eta_{p}=\frac{p \pi}{h}, \quad p=1,2,3 \ldots
\end{aligned}
$$

Applying the finite Fourier transform to equation (4) defined in equation (24) and using the conditions (5)-(11), we get

$$
\frac{\partial^{2} \bar{T}}{\partial r^{2}}+\frac{1}{r} \frac{\partial \bar{T}}{\partial r}+\frac{1}{r^{2}} \frac{\partial^{2} \bar{T}}{\partial \varphi^{2}}-\eta_{p}^{2} \bar{T}=\frac{1}{c} \frac{\partial^{\alpha} \bar{T}}{\partial t^{\alpha}}
$$

the boundary conditions

$$
\begin{aligned}
& k D_{R L}^{I-\alpha} \frac{\partial \bar{T}}{\partial r}=\bar{f}_{1}\left(\varphi, \eta_{p}, t\right), \quad \text { at } r=a, \quad \mathrm{t}>0 \\
& k D_{R L}^{I-\alpha} \frac{\partial \bar{T}}{\partial r}=\bar{f}_{2}\left(\varphi, \eta_{p}, t\right), \quad \text { at } \varphi=0, \quad \mathrm{t}>0 \\
& k D_{R L}^{I-\alpha} \frac{\partial \bar{T}}{\partial r}=\bar{f}_{3}\left(\varphi, \eta_{p}, t\right), \quad \text { at } \varphi=\varphi_{0}, \mathrm{t}>0
\end{aligned}
$$

with

$$
\begin{aligned}
& \bar{T}=0, \quad \text { at } t=0, \quad 0<\alpha<1, \\
& \frac{\partial \bar{T}}{\partial t}=0, \quad \text { at } t=0, \quad 1<\alpha<2,
\end{aligned}
$$

Secondly, we define finite Fourier transform and its inverse transform over the variable $\varphi$ in the range $0 \leq \varphi \leq \varphi_{0}$ as defined in [25] as

$\overline{\bar{T}}\left(r, v, \eta_{p}, t\right)=\int_{\varphi^{\prime}=0}^{\varphi_{0}} K_{0}\left(v, \varphi^{\prime}\right) \bar{T}\left(r, \varphi^{\prime}, \eta_{p}, t\right) d \varphi^{\prime}$

$\bar{T}\left(r, \varphi, \eta_{p}, t\right)=\sum_{v} K_{0}(v, \varphi) \overline{\bar{T}}\left(r, v, \eta_{p}, t\right)$

where

$K_{0}(v, \varphi)=\sqrt{\frac{2}{\varphi_{0}}} \cos (v \varphi)$

where $v$ are the positive roots of $\sin \left(v \varphi_{0}\right)=0$

i.e. $\quad v=\frac{n \pi}{\varphi_{0}}, n=1,2,3 \ldots$

Applying the finite Fourier transform to equation (28) defined in equation (34) and using the conditions (29)-(33), we get

$\frac{\partial^{2} \overline{\bar{T}}}{\partial r^{2}}+\frac{1}{r} \frac{\partial \overline{\bar{T}}}{\partial r}-\frac{v^{2}}{r^{2}} \overline{\bar{T}}-\eta_{p}^{2} \overline{\bar{T}}=\frac{1}{c} \frac{\partial^{\alpha} \overline{\bar{T}}}{\partial t^{\alpha}}$

with

$k D_{R L}^{I-\alpha} \frac{\partial \overline{\bar{T}}}{\partial r}=\overline{\bar{f}}_{1}\left(v, \eta_{p}, t\right)$, at $r=a, \quad \mathrm{t}>0$

$\overline{\bar{T}}=0$,

at $t=0, \quad 0<\alpha<1$,

$\frac{\partial \overline{\bar{T}}}{\partial t}=0$

at $t=0, \quad 1<\alpha<2$,

Lastly, we define finite Hankel transform and its inverse over the variable $r$ in the range $0 \leq r<a$ as defined in [25] respectively as,

$\overline{\bar{T}}\left(\beta_{m}, v, \eta_{p}, t\right)=\int_{r^{\prime}=0}^{a} r^{\prime} K_{1}\left(\beta_{m}, r^{\prime}\right) \overline{\bar{T}}\left(r^{\prime}, v, \eta_{p}, t\right) d r^{\prime}$

$\overline{\bar{T}}\left(r, v, \eta_{p}, t\right)=\sum_{m=1}^{\infty} K_{1}\left(\beta_{m}, r\right) \overline{\bar{T}}\left(\beta_{m}, v, \eta_{p}, t\right)$

where

$$
K_{1}\left(\beta_{m}, r\right)=\frac{\sqrt{2}}{a} \frac{1}{\left[1-\frac{v^{2}}{\beta_{m} a^{2}}\right]^{1 / 2}} \frac{J_{0}\left(\beta_{m} r\right)}{J_{0}\left(\beta_{m} a\right)}
$$


and $\beta_{1}, \beta_{2}, \ldots$ are the positive roots of the transcendental equation

$$
J_{1}\left(\beta_{m} a\right)=0, \quad m=1,2,3 \ldots
$$

Applying the finite Hankel transform to equation (38) defined in equation (42) and using the conditions (39)-(41), we get

$\frac{\partial^{\alpha} \overline{\bar{T}}\left(\beta_{m}, v, \eta_{p}, t\right)}{\partial t^{\alpha}}+c\left(\beta_{m}^{2}+\frac{v^{2}}{r^{2}}+\eta_{p}^{2}\right) \overline{\bar{T}}\left(\beta_{m}, v, \eta_{p}, t\right)=A\left(\beta_{m}, v, \eta_{p}, t\right)$

with

$\overline{\bar{T}}\left(\beta_{m}, \nu, \eta_{p}, t\right)=0, \quad$ for $t=0, \quad 0<\alpha<1$,

$\frac{\partial \overline{\bar{T}}\left(\beta_{m}, v, \eta_{p}, t\right)}{\partial t}=0, \quad$ for $t=0, \quad 1<\alpha<2$,

where

$$
\begin{aligned}
& A\left(\beta_{m}, v, \eta_{p}, t\right)=c a K_{1}\left(\beta_{m}, a\right) \overline{\bar{f}}_{1}\left(v, \eta_{p}, t\right) \\
& \left\{\left.a \frac{d K_{0}(v, \varphi)}{d \varphi} \overline{\bar{f}}_{2}\left(\beta_{m}, \eta_{p}, t\right)\right|_{\varphi=0}-\left.\frac{d K_{0}(v, \varphi)}{d \varphi} \overline{\bar{f}}_{3}\left(\beta_{m}, \eta_{p}, t\right)\right|_{\varphi=\varphi_{0}}\right. \\
& \left.+\left.\frac{d K\left(\eta_{p}, z\right)}{d z} \overline{\bar{f}}_{4}\left(\beta_{m}, v, t\right)\right|_{z=0}+\left.\frac{d K\left(\eta_{p}, z\right)}{d z} \overline{\bar{f}}_{5}\left(\beta_{m}, v, t\right)\right|_{z=h}\right\}
\end{aligned}
$$

Applying the Laplace transform and their inverse to equation (46), we get

$$
\overline{\bar{T}}\left(\beta_{m}, v, \eta_{p}, t\right)=\frac{A\left(\beta_{m}, v, \eta_{p}, t\right)}{c\left(\beta_{m}^{2}+\eta_{p}^{2}\right)}\left[1-E_{\alpha}\left(-k\left(\beta_{m}^{2}+\frac{v^{2}}{r^{2}}+\eta_{p}^{2}\right) t^{\alpha}\right)\right]
$$

Finally, taking the inverses defined in (43), (35) and (25) of equation (50), we obtain the required temperature as:

$$
\begin{aligned}
& T(r, v, z, t)=\sum_{m=1}^{\infty} \sum_{v} \sum_{p=1}^{\infty} K_{1}\left(\beta_{m}, r\right) K_{0}(v, \varphi) K\left(\eta_{p}, z\right) \frac{1}{c\left(\beta_{m}^{2}+\frac{v^{2}}{r^{2}}+\eta_{p}^{2}\right)} \\
& {\left[1-E_{\alpha}\left(-k\left(\beta_{m}^{2}+\frac{v^{2}}{r^{2}}+\eta_{p}^{2}\right) t^{\alpha}\right)\right] \times b_{m n} }
\end{aligned}
$$

where

$$
\begin{aligned}
b_{m n} & =\left\{c \cdot a \cdot K_{1}\left(\beta_{m}, a\right) \int_{\varphi^{\prime}=0}^{\varphi_{0}} \int_{z^{\prime}=0}^{h} K_{0}\left(v, \varphi^{\prime}\right) K\left(\eta_{p}, z^{\prime}\right) \cdot f_{1}\left(\varphi^{\prime}, z^{\prime}, t^{\prime}\right) \cdot d \varphi^{\prime} d z^{\prime}\right. \\
& +c \cdot v \cdot \sqrt{\frac{2}{\varphi_{0}}} \int_{r^{\prime}=0}^{a} \int_{z^{\prime}=0}^{h} r^{\prime} K_{1}\left(\beta_{m}, r^{\prime}\right) K\left(\eta_{p}, z^{\prime}\right) \cdot f_{2}\left(r^{\prime}, z^{\prime}, t^{\prime}\right) \cdot d r^{\prime} d z^{\prime} \\
& -c . v \cdot \sqrt{\frac{2}{\varphi_{0}}} \cos \left(v \varphi_{0}\right) \int_{r^{\prime}=0}^{a} \int_{z^{\prime}=0}^{h} r^{\prime} K_{1}\left(\beta_{m}, r^{\prime}\right) K\left(\eta_{p}, z^{\prime}\right) \cdot f_{3}\left(r^{\prime}, z^{\prime}, t^{\prime}\right) \cdot d r^{\prime} d z \\
& +\sqrt{\frac{2}{\pi}} c \cdot \eta_{p} \cdot \int_{r^{\prime}=0}^{a} \int_{\varphi^{\prime}=0}^{\varphi_{0}} r^{\prime} K_{1}\left(\beta_{m}, r^{\prime}\right) K_{0}\left(v, \varphi^{\prime}\right) \cdot f_{4}\left(r^{\prime}, z^{\prime}, t^{\prime}\right) \cdot d r^{\prime} d \varphi^{\prime} \\
& \left.+\sqrt{\frac{2}{\pi}} c \cdot \eta_{p} \cdot \cos \left(\eta_{p} h\right) \cdot \int_{r^{\prime}=0}^{a} \int_{\varphi^{\prime}=0}^{\varphi_{0}} r^{\prime} K_{1}\left(\beta_{m}, r^{\prime}\right) K_{0}\left(v, \varphi^{\prime}\right) \cdot f_{5}\left(r^{\prime}, z^{\prime}, t^{\prime}\right) \cdot d r^{\prime} d \varphi^{\prime}\right)
\end{aligned}
$$

$$
K_{1}\left(\beta_{m}, r\right)=\frac{\partial}{\partial r}\left[K_{0}\left(\beta_{m}, r\right)\right]
$$

Using equation (51) in equation (17) the displacement function $\psi$, as follows:

$$
\begin{aligned}
\frac{\partial^{2} \psi}{\partial r^{2}}+\frac{1}{r} & \frac{\partial \psi}{\partial r}=(1+v) a_{t} \sum_{m=1}^{\infty} \sum_{v} \sum_{p=1}^{\infty} K_{1}\left(\beta_{m}, r\right) K_{0}(v, \varphi) K\left(\eta_{p}, z\right) \\
& \frac{1}{c\left(\beta_{m}^{2}+\frac{v^{2}}{r^{2}}+\eta_{p}^{2}\right)}\left[1-E_{\alpha}\left(-k\left(\beta_{m}^{2}+\frac{v^{2}}{r^{2}}+\eta_{p}^{2}\right) t^{\alpha}\right)\right] \times b_{m n}
\end{aligned}
$$

Equation (54) becomes:

$$
\begin{aligned}
\psi= & -(1+v) a_{t} \sum_{m=1}^{\infty} \sum_{v} \sum_{p=1}^{\infty} \frac{1}{\beta_{m}^{2}} K_{1}\left(\beta_{m}, r\right) K_{0}(v, \varphi) K\left(\eta_{p}, z\right) \\
& \frac{1}{c\left(\beta_{m}^{2}+\frac{v^{2}}{r^{2}}+\eta_{p}^{2}\right)}\left[1-E_{\alpha}\left(-k\left(\beta_{m}^{2}+\frac{v^{2}}{r^{2}}+\eta_{p}^{2}\right) t^{\alpha}\right)\right] \times b_{m n}
\end{aligned}
$$

Using equation (55) in equations (19)-(20) we obtain the thermal stresses as:

$$
\begin{array}{r}
\sigma_{r r}=-2(1+v) a_{t} \mu \sum_{m=1}^{\infty} \sum_{v} \sum_{p=1}^{\infty} \frac{1}{r \beta_{m}} K_{2}\left(\beta_{m}, r\right) K_{0}(v, \varphi) K\left(\eta_{p}, z\right) \\
\frac{1}{c\left(\beta_{m}^{2}+\frac{v^{2}}{r^{2}}+\eta_{p}^{2}\right)}\left[1-E_{\alpha}\left(-k\left(\beta_{m}^{2}+\frac{v^{2}}{r^{2}}+\eta_{p}^{2}\right) t^{\alpha}\right)\right] \times b_{m n}
\end{array}
$$

$$
\begin{gathered}
\sigma_{\theta \theta}=-2(1+v) a_{t} \mu \sum_{m=1}^{\infty} \sum_{v} \sum_{p=1}^{\infty} \frac{1}{\beta_{m}}\left(\beta_{m} K_{2}\left(\beta_{m}, r\right)-\frac{K_{2}\left(\beta_{m}, r\right)}{r}\right) K_{0}(v, \varphi) \\
K\left(\eta_{p}, z\right) \frac{1}{c\left(\beta_{m}^{2}+\frac{v^{2}}{r^{2}}+\eta_{p}^{2}\right)}\left[1-E_{\alpha}\left(-k\left(\beta_{m}^{2}+\frac{v^{2}}{r^{2}}+\eta_{p}^{2}\right) t^{\alpha}\right)\right] \times b_{m n}
\end{gathered}
$$

where

$$
K_{2}\left(\beta_{m}, r\right)=\frac{\partial}{\partial r}\left[K_{1}\left(\beta_{m}, r\right)\right]
$$

\section{Numerical Results and Discussion}

Setting

$$
\begin{aligned}
& f_{1}(\varphi, z, t)=\left(\varphi^{2}-\varphi_{0}^{2}\right)^{2}\left(z^{2}-h^{2}\right)^{2} e^{-A t} \\
& f_{2}(\varphi, z, t)=\left(r^{2}-a^{2}\right)^{2}\left(z^{2}-h^{2}\right)^{2} e^{-A t} \\
& f_{3}(\varphi, z, t)=\left(r^{2}-a^{2}\right)^{2}\left(z^{2}-h^{2}\right)^{2} e^{-A t} \\
& f_{4}(\varphi, z, t)=\left(r^{2}-a^{2}\right)^{2}\left(\varphi^{2}-\varphi_{0}^{2}\right)^{2} e^{-A t} \\
& f_{5}(\varphi, z, t)=\left(r^{2}-a^{2}\right)^{2}\left(\varphi^{2}-\varphi_{0}^{2}\right)^{2} e^{-A t} \\
& \text { where } r \text { is the radius and } A>0 .
\end{aligned}
$$

\subsection{Dimension}

Radius of a circular sector disk $a=1 \mathrm{~m}$,

$\varphi^{\top}$ Thickness of circular sector disk $h=0.1 \mathrm{~m}$
Portion of circular sector disk $\varphi_{0}=270^{\circ}$ 
Central circular path of disk in radial and axial directions: $r_{l}=1 \mathrm{~m}$, and $z_{l}=0.05 \mathrm{~m}$ and $\varphi_{0}=135^{\circ}$.

The first five positive root of the transcendental equation $J_{1}\left(\beta_{m} a\right)=0$ as defined in [25] are $\beta_{1}=3.8317$, $\beta_{2}=7.0156, \beta_{3}=10.1735, \beta_{4}=13.3237, \beta_{5}=16.470$. The pure aluminum material was chosen for purposes of numerical evaluations and the constants of the problem were taken as following table 1 .

Table 1. Material Constants.

\begin{tabular}{ll}
\hline Physical constants & Value \\
\hline Thermal diffusivity $(\mathrm{c})$ & $84.18 \mathrm{~m}^{2} / \mathrm{s}$ \\
Thermal conductivity $(\mathrm{k})$ & $204 \mathrm{~W} /(\mathrm{m} . \mathrm{K})$ \\
Young's modulus $(\mathrm{E})$ & $70 \mathrm{GPa}$ \\
Coefficient of thermal expansion $\left(a_{t}\right)$ & $22.2 \times 10^{-6} \mathrm{~K}^{-1}$ \\
Specific heat $\left(c_{p}\right)$ & $896 \mathrm{~J} /(\mathrm{kg} . \mathrm{K})$ \\
Lame's constants $(\mu)$ & $26.67 \mathrm{GPa}$ \\
Poisson's ratio $(v)$ & 0.35 \\
Density $(\rho)$ & $2707 \mathrm{~kg} / \mathrm{m}^{3}$ \\
\hline
\end{tabular}

The numerical calculation and graphs have been carried out with help of computational mathematical software [26] PTC Mathcad Prime-6.0. Figure 2-5 shows the variation of temperature, displacement, stresses in radial distance $r$ at instants $\alpha=0.50$ for time parameter $t=0.25,0.50,0.75,1$. From figure 2 . The temperature profile increases within the region $0 \leq r \leq 0.4,0.7 \leq r \leq 1$ and decreases within the region $0.4 \leq r \leq 0.7$ in the radial direction. Figure 3 . indicates the variation of displacement along the radial direction, it is clear that displacement decreases within the region $0 \leq r \leq 0.4,0.7 \leq r \leq 1$ and increases within the region $0.4 \leq r \leq 0.7$ with increasing the radius. Figure 4 . Shows the radial stress distribution, it is observed that stress distribution decreases within the region $0 \leq r \leq 0.5$, $0.8 \leq \mathrm{r} \leq 1$ and increases within the region $0.5 \leq r \leq 0.8$ with increasing the radius. Figure 5. indicates the circumferential stress distribution in the radial direction, it is observed that it will decrease in the region $0 \leq r \leq 0.2$, $0.5 \leq r \leq 0.8$ and increase within the region $0.2 \leq r \leq 0.5$, $0.8 \leq r \leq 1$.

Figure 6-9, depicts the temperature, displacement, and stresses in radial direction at time $t=0.50$ for different values of $\alpha=0.5,1,1.5,2$. From Figure 6 . We can see that temperature distribution increases in the region $0 \leq r \leq 0.4$, $0.7 \leq \mathrm{r} \leq 1$ and decreases within the region $0.4 \leq r \leq 0.7$ with the value of $\alpha$ increase with increasing the radial distance. Figure 7. indicates the displacement in the radial direction with different fractional-order parameters, it is clear that it will decrease in the region $0 \leq r \leq 0.4$, $0.7 \leq r \leq 1$ and increase within the region $0.4 \leq r \leq 0.7$ with the value of $\alpha$ increase. From Figure 8. the radial stress distribution decreases with increasing the value of $\alpha$ in the region $0 \leq r \leq 0.5,0.8 \leq r \leq 1$ and increases within the region $0.5 \leq r \leq 0.8$ with increasing the radius. Figure 9 . indicates the circumferential stress distribution, it will decrease with increasing the $\alpha$ in the region $0 \leq r \leq 0.2$, $0.5 \leq r \leq 0.8$ and increase in the region $0.2 \leq r \leq 0.5$, $0.8 \leq r \leq 1$ in the radial direction.

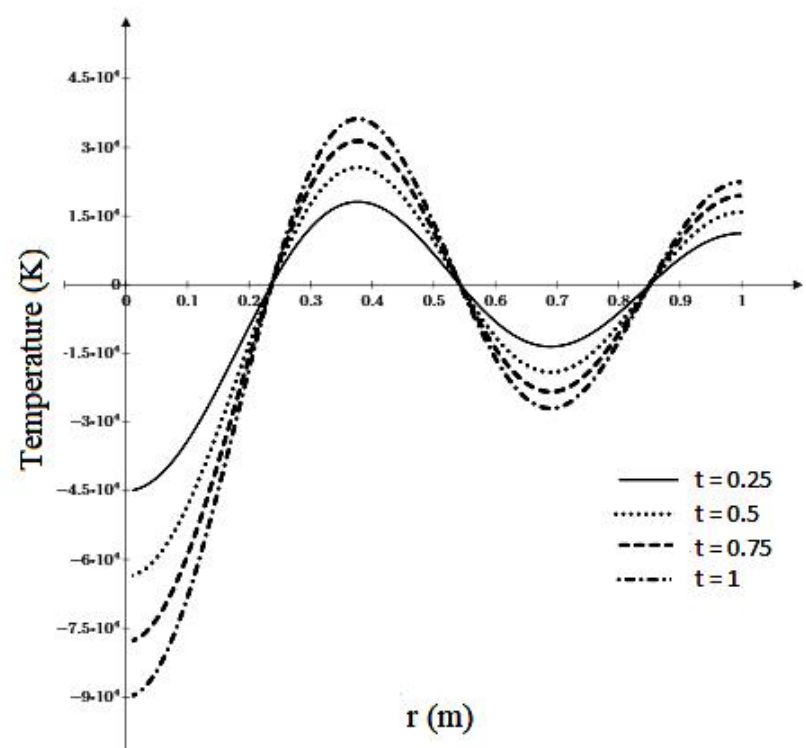

Figure 2. Temperature distribution at $\alpha=0.5$ and different values of $t$.

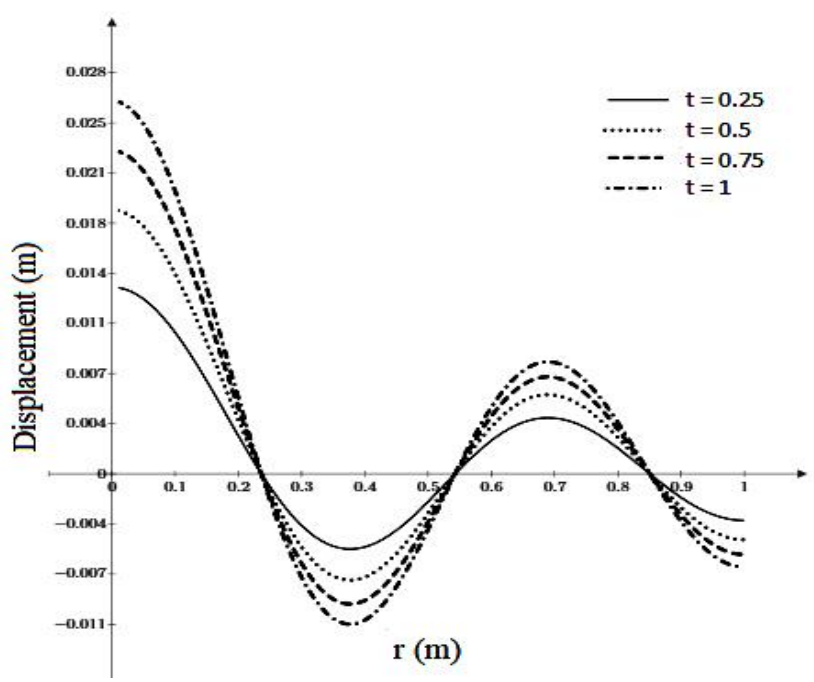

Figure 3. Displacement distribution at $\alpha=0.5$ and different values of $t$.

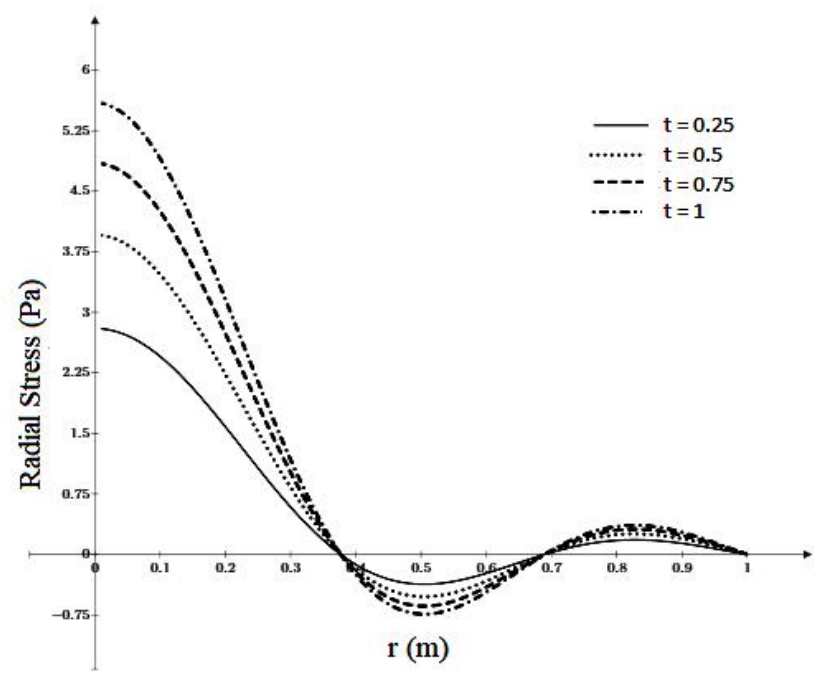

Figure 4. Radial stress distribution at $\alpha=0.5$ and different values of $t$. 


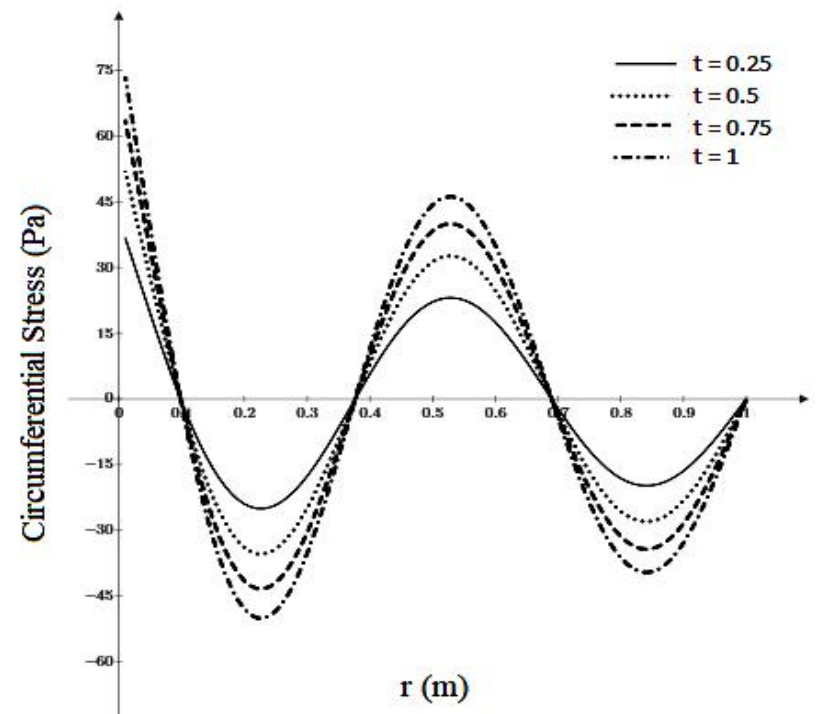

Figure 5. Circumferential stress distribution at $\alpha=0.5$ and different values of $t$.

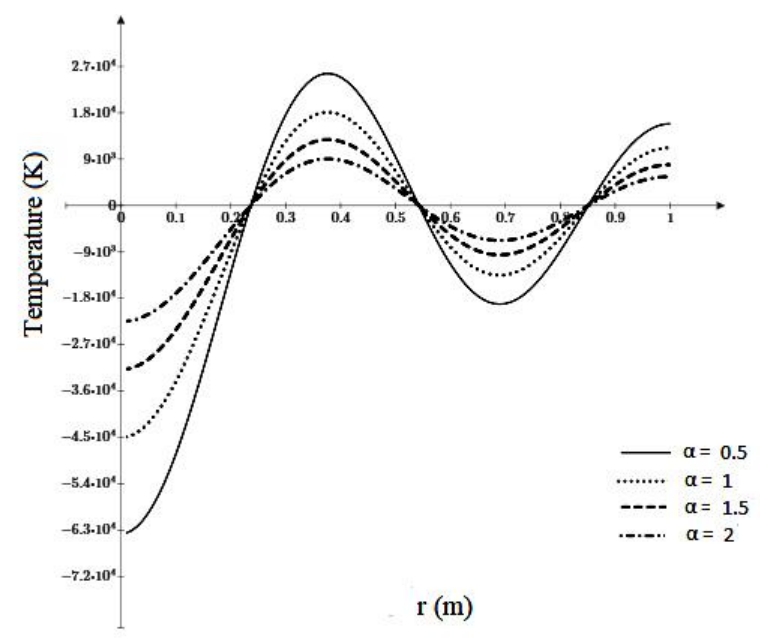

Figure 6. Temperature distribution at $t=0.5$ and different values of $\alpha$.

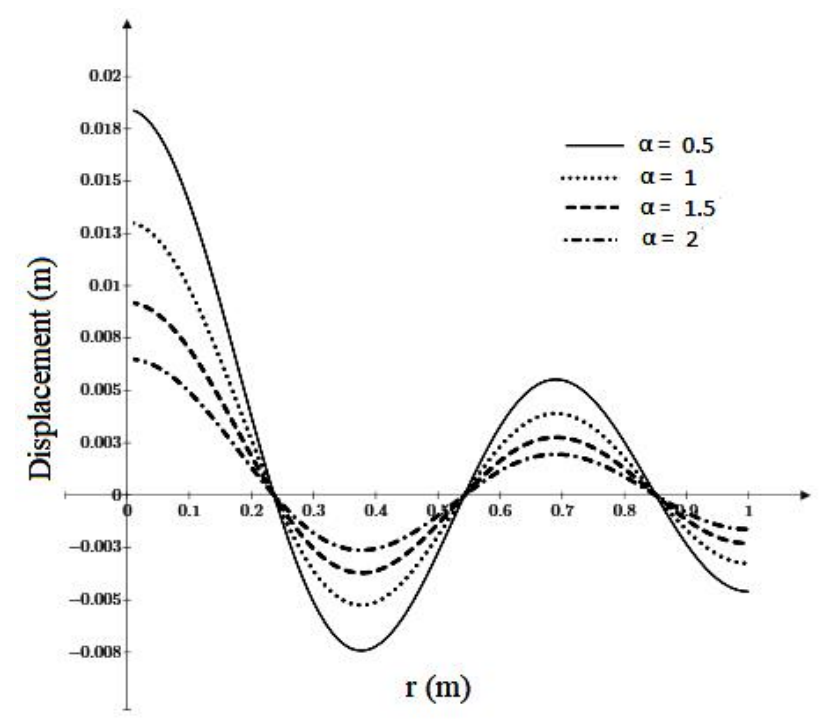

Figure 7. Displacement distribution at $t=0.5$ and different values of $\alpha$.

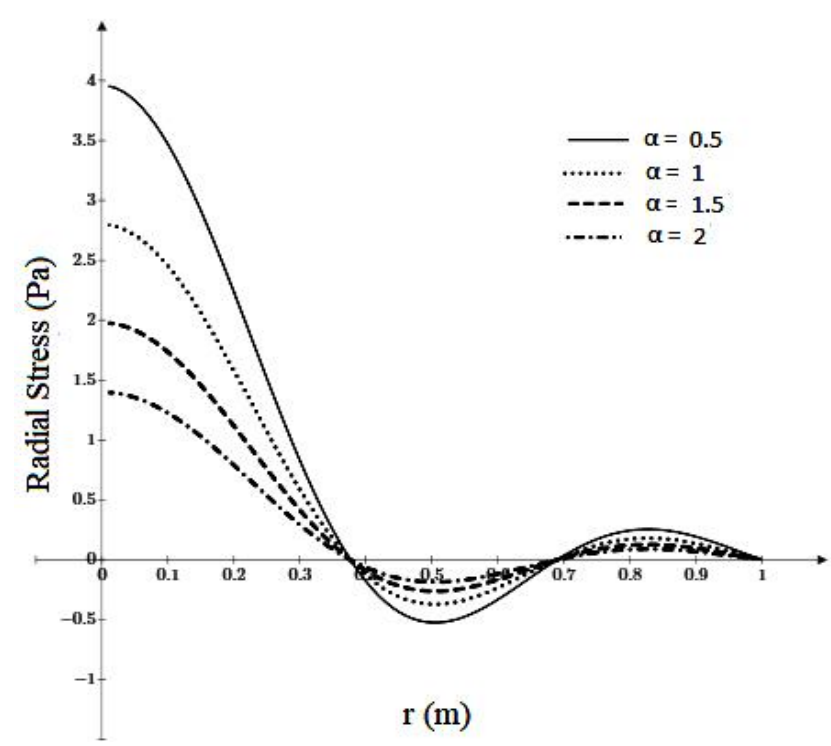

Figure 8. Radial stress distribution at $t=0.5$ and different values of $\alpha$.

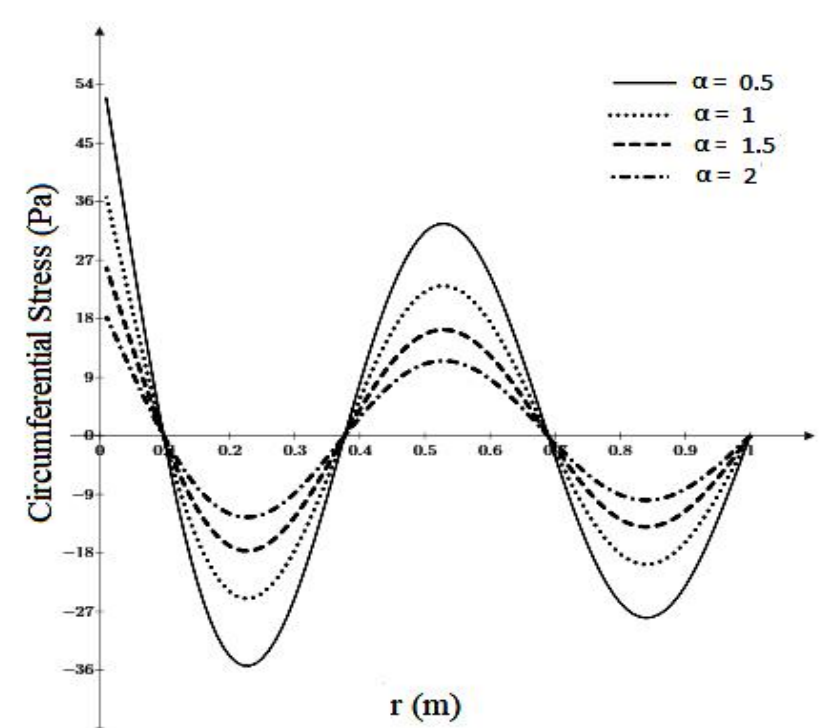

Figure 9. Circumferential stress distribution at $t=0.5$ and different values of $\alpha$.

\section{Conclusion}

This article analyzed the temperature, displacement, and thermal stresses for a circular sector disk occupying the space $0 \leq r \leq a, 0 \leq \varphi \leq \varphi_{0} \leq 2 \pi, 0 \leq z \leq h$. The general solution obtained from finite Hankel, Fourier, and Laplace transform and its inverses. Figure 2-5, shows the temperature, displacement, and thermal stresses at $\alpha=0.5$ for times $t=0.25,0.50,0.75,1$.

1. From the figures of temperature and displacement, we observed that the direction of heat flow and direction of body displacement are opposite and they are inversely proportionally to each other.

2. From the figures of radial and circumferential stresses, it develops the tensile and compressive stresses in the radial direction.

3. The fractional-order parameter $0<\alpha<1$, $1<\alpha<2$ and $\alpha=1$ indicates the weak, strong, and normal conductivity respectively.

4. From figures 6-9, we see that the timefractional derivatives play a significant role in all quantities in the given field and changes in 
the values of the parameter $\alpha$. This work may prove useful in material science, designers, real-life engineering problems, physicists, and those working to further develop the theory of fractional-order thermoelasticity.

\section{Acknowledgements:}

The authors are grateful thanks to Chhatrapati Shahu Maharaja Research, Training and Human Development Institute (SARTHI) for awarding the Chief Minister Special Research Fellowship - 2019 (CMSRF - 2019).

\section{Nomenclature (List of Symbols):}

$$
\begin{array}{ll}
a_{t} & \text { linear coefficient of thermal expansion }\left(\mathrm{K}^{-1}\right) \\
c & \text { thermal diffusivity }\left(\mathrm{m}^{2} \cdot \mathrm{s}^{-1}\right) \\
c_{p} & \text { specific heat at constant strain }(\mathrm{J} / \mathrm{Kg} \cdot \mathrm{K}) \\
E & \text { cubical dilation }\left(\mathrm{K}^{-1}\right) \\
k & \text { thermal conductivity }(\mathrm{W} / \mathrm{m} . \mathrm{K}) \\
T & \text { absolute temperature }(\mathrm{K}) \\
U_{i} & \text { Components of displacement vector }(\mathrm{m})
\end{array}
$$

\section{Greek symbols}

$\begin{array}{cl}\mu & \text { Lame's constants }(\mathrm{GPa}) \\ v & \text { Poisson's ratio } \\ \rho & \text { density }\left(\mathrm{kg} / \mathrm{m}^{3}\right) \\ \psi & \text { displacement potential function } \\ \delta_{\mathrm{ij}} & \text { Kronecker's delta tensor } \\ \sigma_{\mathrm{ij}} & \text { components of stress tensor } \\ \sigma_{\mathrm{rr}} & \text { radial stress }(\mathrm{Pa}) \\ \sigma_{\theta \theta} & \text { circumferential stress }(\mathrm{Pa})\end{array}$

\section{Abbreviations}

$$
\begin{aligned}
& 1 D \text { one-dimensional (m) } \\
& 2 D \text { two-dimensional (m) } \\
& 3 D \text { three-dimensional (m) }
\end{aligned}
$$

\section{References:}

[1] H. W. Lord, Y. Shulman, "A Generalized Dynamical Theory of Thermoelasticity," J. Mech. Phys. Solids., 15, 299-307, 1967.

[2] A. Green, P. Naghdi, "Thermoelasticity Without Energy Dissipation,” J. Elasticity., 31, 189-208, 1993.

[3] Y. Ootao, T. Akai, Y. Tanigawa, “Three Dimentional Transient Thermal Stress Analysis of a Nonhomogeneous Hollow Circular Cylinder Due to a Moving Heat Source in the Axial Direction," Journal of Thermal Stresses., 18, 497-512, 1995.

[4] M. Ishihrar, N. Noda, "Theoretical Analysis of Thermoelastoplastic Deformation of a Circular Plate Due to a Partially Distributed Heat Supply," Journal of Thermal stresses., 20, 203-225, 1997.

[5] H. Sherief, F. Megahed, "A Two-Dimensional Thermoelasticity Problem for a Half-Space Subjected to
Heat Sources,” Int. J. Solids Structures., 36, 1369-1382, 1999.

[6] I .Podlubny, "Geometric and Physical Interpretation of Fractional Integration and Fractional Differentiation," Fractional Calculus and Applied Analysis., 4, 367-386, 2002.

[7] Y. Z. Povstenko, "Fractional Heat Conduction Equation and Associated Thermal Stress," Journal of Thermal Stresses., 28, 83-102, 2005.

[8] H. H.Sherief, H .A. Saleh, "A Half-Space Problem in the Theory of Generalized Thermoelastic Diffusion," Int. J. Solids. Struct., 42, 4484 - 4494, 2005.

[9] Y. Z. Povstenko, "Thermoelasticity that uses Fractional Heat Conduction Equation," J. Math. Sci., 162, 296305, 2009.

[10] H. H.Sherief, A.El-Said, A. Abd El-Latief, "Fractional order Theory of Thermoelasticity," International Journal of Solids and Structures., 47, 269-275, 2010.

[11] K. R. Gaikwad, K. P. Ghadle, "Quasi-Static Thermoelastic Problem of an Infinitely Long Circular Cylinder," Journal of the Korean Society for Industrial and Applied Mathematics., 14, 141-149, 2010.

[12] A. S. El-Karamany, M. A. Ezzat, "On Fractional Thermoelasticity," Mathematics and Mechanics of Solids., 16, 334-346, 2011.

[13] A. Sur, M. Kanoria, "Fractional order TwoTemperature Thermoelasticity with Wave Speed," Acta Mechanica., 223, 2685-2701, 2012.

[14] K. R. Gaikwad, K.. P. Ghadle, "Nonhomogeneous Heat Conduction Problem and its Thermal Deflection Due to Internal Heat Generation in a Thin Hollow Circular Disk," Journal of Thermal stresses., 35, 485498, 2012.

[15] K. R. Gaikwad, K. P. Ghadle, "On a Certain Thermoelastic Problem of Temperature and Thermal Stresses in a Thick Circular Plate," Australian Journal of Basic and Applied Sciences., 6, 34-48, 2012.

[16] A. Sur, M. Kanoria, "Fractional order Generalized Thermoelastic Functionally Graded Solid with Variable Material Properties," Journal of Solid Mechanics., 6, 54-69, 2014.

[17] W. Raslan, "Application of Fractional order Theory of Thermoelasticity To A 1D Problem For A Cylindrical Cavity," Arch. Mech., 66, 257-267, 2014.

[18] W. Raslan, “Application of Fractional order Theory of Thermoelasticity in a Thick Plate Under Axisymmetric Temperature Distribution," Journal of Thermal Stresses., 38, 733-743, 2015.

[19] K. R. Gaikwad, "Mathematical Modelling of Thermoelastic Problem in a Circular Sector Disk Subject to Heat Generation," International Journal of Advances in Applied Mathematics and Mechanics., 3, 183-195, 2015.

[20] K. R. Gaikwad, "Two-Dimensional Study-State Temperature Distribution of a Thin Circular Plate Due to Uniform Internal Energy Generation," Cogent Mathematics, Taylor and Francis Group, 3, 1-10, 2016. 
[21] K. R. Gaikwad, “Axi-Symmetric Thermoelastic Stress Analysis of a Thin Circular Plate Due to Heat Generation," International Journal of Dynamical Systems and Differential Equations., 9, 187-202, 2019.

[22] K. R. Gaikwad, S. G. Khavale, "Time Fractional Heat Conduction Problem of a Thin Hollow Circular Disk And It'S Thermal Deflection," Easy Chair Preprint., 1672, 1-11, 2019.

[23] I. Podlubny, Fractional Differential Equation, Academic Press, San Diego, 1999.

[24] I. N. Sneddon, The use of Integral Transform, McGraw Hill, New York, 1972.
[25] N. M. Ozisik, Boundary Value Problem of Heat Conduction, International Textbook Company, Scranton, Pennsylvania, 84-101, 1968.

[26] PTC Mathcad Prime-6.0.0.0, [Online]. Available: https://support.ptc.com/help/mathcad/r6.0/en/ (accessed Nov. 1, 2020). 